Keywords: Demoralization; Problem based learning; Medical student education; DSM.

\title{
Teaching medical students to recognize demoralization
}

\author{
Julia B. Frank, MD \\ Department of Psychiatry and Behavioral \\ Sciences, George Washington University \\ School of Medicine and Health \\ Sciences, Washington, DC
}

USA

\begin{abstract}
Background and Objectives: Concepts such as demoralization fit well into Problem or Case-based learning methods that encourage students to organize knowledge based on clinical problems, rather than according to the disciplines of basic science.

Methods: At two US schools, psychiatry clerkship students learn about demoralization and psychotherapy through structured, case based exercises that teach them to elicit and respond to patients' life stories in ways that emphasize hope and empowerment.

Results: Students' reactions to these exercises, though mixed, suggest that they may enhance students' understanding of the universal elements of distress (demoralization) that cut across many disabling conditions and of the role that caregivers may play in compounding or relieving this distress.

Conclusions: Learning to recognize and respond to demoralization is an advanced communication skill that can be introduced during a psychiatry clerkship.
\end{abstract}

\section{Introduction}

Medicine since the time of Virchow requires practitioners to relate observed symptoms and signs to the underlying condition of organs, tissues, cells, and now genes. Medical students, especially in Eurowestern countries, study psychiatric disorders by drawing analogies to other diseases. A concept like demor- alization, uniting a wide range of phenomena without anchoring them in measurable physiological processes, fits into contemporary medicine about as well as humors fit into medicine in the golden age of pathology.

Teaching students and clinicians to recognize, think about and respond to demoralization requires weaning them from the apparently clear and concrete criteria of the Diagnostic 
and Statistical Manual of Mental Disorders IV-TR (DSM IV-TR) ${ }^{1}$ that currently dominates psychiatric education. Students must also overcome their squeamishness about pathologizing normal distress. Tact and empathy, two praiseworthy qualities in doctor patient interaction may, at times, blind carers to important phenomena. Students rightly hesitate to apply a possibly stigmatizing label to people who have already suffered the calamity of having a serious medical/psychiatric condition.

Educating medical students about demoralization in the medically ill, furthermore, threatens their growing professionalism. Understanding the concept means acknowledging that the medical system, and more particularly, the behavior of admired actors within that system, may contribute to patients' distress. Students' intense focus on acquiring medical knowledge and completing clinical tasks diverts their attention from how they and those they wish to imitate may contribute to patients' suffering.

Fortunately, the paradigm of medical education is shifting away from teaching students facts and theories, then exposing them to patients as examples that prove the validity of the basic science they have been taught. New ways of annealing science with clinical reality include the methods of learning that begin with a patient (Case Based Learning or $\mathrm{CBL})^{2}$ or a problem (Problem Based Learning or $\mathrm{PBL})^{3}$. In a CBL exercise, in addition to clues about the patient's disease, students receive background personal and social information that they must relate to the progress and outcome of the case. At various points during the discussion, students still consult textbooks to understand the physiology and pathology associated with particular symptoms, but they must also relate individual cases to broad domains such as epidemiology, the range of clinical management op- tions, various possible outcomes, and different presentations of illness in different cultures or stages of personal development.

Students in clinical settings are inevitably immersed in CBL. Real life experience confronts them with patients' social needs and emotional states. Demoralization, which by definition is both a quality of the person and a response to particular situations, fits well into the framework of CBL.

\section{Introducing demoralization and psychotherapy during the psychiatry clerkship}

At the George Washington University School of Medicine, clerkship students work mostly with inpatients. We combine didactic instruction, self-guided learning and an assigned clinical exercise to introduce the concept of demoralization in the context of providing inpatient psychiatric care. This learning is reinforced by senior psychiatrists and residents, who routinely focus on the state of patients' morale in their clinical work. This paper describes the exercise we use with third year clerks.

At the beginning of the clerkship, students learn to take a focused developmental history from their patients. The interview protocol involves questions about patients' birth, early family life, family cultural background, medical experiences, substance use, school and work history and important relationships over time. The problems uncovered in the developmental history go beyond the symptoms and signs required for diagnosis. Students quickly recognize the need for treatment, not just for symptom resolution, but also to help patients make sense of their life experience in ways that open up avenues of hope for themselves going forward. Psychotherapy becomes the obvious answer to the question of "what does this patient really need?". 
After the introductory session, students are directed to a one page description of psychotherapy as a particular relationship whose ultimate purpose is to instill hope and encourage self- efficacy in patients, in order to relieve demoralization ${ }^{4}$. Psychotherapy is presented, not as an arcane practice that occurs in formal settings students will never see, but as a mode of communication that may be woven into routine, day to day interactions. Any interaction that encourages hope and self-efficacy or mastery is presented as a legitimate analog to psychotherapy ("therapeutic communication"). Students are also encouraged to recognize that discouraging or disempowering ("counter-therapeutic") communication may become a source of apparent psychopathology, by engendering demoralization.

Following the introduction, students work through a self- guided exercise available to them on the course website. The exercise involves analyzing four case scenarios, a practice they are familiar with from many other elements of the general curriculum. Two of the scenarios illustrate medical encounters and two portray interactions with psychiatric patients that resemble the ones they are seeing on the wards.

General medical example

A healthy 30 year old woman visits a neurologist for recurrent headaches that sound like classic migraines with multiple triggers: menstrual cycle, diet, stress and sleep. She has been given propranolol for prevention, which has helped, but the headaches still recur and can be disabling. "Just to be sure" the neurologist orders an MRI.

Patient: "Did you get a report from the MRI? Being in that machine was pretty scary".

Neurologist: "I am glad to tell you the MRI showed no signs of tumors or vascu- lar malformations. You should live a long life. Just keep on taking the propranolol. You don't need to follow up with me. Sorry this took so long and was so unpleasant. You probably didn't really need a neurologist in the first place!".

Psychiatric Example:

A 25 year old patient with depression is admitted following a suicide attempt. $\mathrm{He}$ grew up in a family in which his alcoholic stepfather beat him and his mother was depressed. He dropped out of high school but later got his GED. His girlfriend broke up with him because he didn't want to assume responsibility for her two young children. In his view, without more education, he can barely take care of himself. She didn't understand that he was planning to try to find a better job. He feels rejected and hurt, and believes her rejection proves that women can't be trusted. Since the one thing that he hopes for is to have a close relationship, this belief seems to justify his suicidal act.

Patient: "I can't get any rest, I keep thinking about Carla. I miss her and I just can't understand how she could dump me like that. I'm losing weight, I can't concentrate at work, nothing makes me feel better."

Doctor: "I think your symptoms are bad enough that you should take medication for them. An SSRI works really well for helping people sleep and be less agitated. The side effects aren't bad, either. We should start that tomorrow. And I'll also give you something for sleep, just until you start to respond to it".

At the end of each vignette, students are prompted to list

Therapeutic elements:

Counter-therapeutic elements:

After reading and responding to four vignettes, students scroll down to compare their 
answers with the ones provided by the course director. The answers to the two vignettes are:

Therapeutic elements (headache with normal MRI):

- Relief of anxiety about ominous possibilities.

- Doctor expresses empathy for the unpleasantness of the test procedure.

Counter therapeutic elements (and how they could be modified):

- Neurologist makes patient feel like she shouldn't have come (non-acceptance). The doctor could have said "It was a good idea to get this checked out" before giving the results.

- Patient leaves without any idea of what or who can help with the headaches she still has (no empowerment). The neurologist could have suggested that she ask her primary care doctor about other types of prevention, that she look for self-help materials for migraine sufferers, etc.

Therapeutic elements (depression).

- Doctor offers realistic help, with empathic concern for side effects and target symptoms.

- Counter- therapeutic elements

- Doctor's empathy is limited. Not acknowledging the reality of the patient's loss and hurt may silently confirm to him that the situation is hopeless. (Alternative: "I know you feel really hurt and hopeless, and that you are also tormented by not sleeping and not being ever able to feel any relief. I think if I give you medication that helps you function better, your situation won't look so bad to you as it does now").

- Sometimes it is important to challenge a patient's experience as well as empathize with it. ("I know how much she meant to you, though I can also see how a moth- er of young children might need someone to help her care for them and put their needs first, even if she cared about you. That doesn't mean all women are untrustworthy, or that no one will ever care about you").

By offering concrete, plausible alternatives to counter-therapeutic communication, the analyses of the vignettes are meant to empower rather than discourage students. Students tend to see psychotherapy as exotic and inordinately time consuming. The implication of these examples is that therapeutic interactions may be very brief and need not require extensive training.

Whether or not students do the self-guided, preparatory exercise, all are required to ask a resident or attending physician to help them select an inpatient with whom to apply the principles of therapeutic communication. They are instructed to choose a time and place to elicit a full developmental and social history from the patient. While similar to the generic history taught as part of initial assessment, other specific questions ${ }^{5}$ are suggested, to uncover sources of demoralization and resilience. These include

- What was the best year of your life, and why?

- What was the most challenging year of your life, and why?

- What are some of the things you have done in your life that you are most proud of?

- What is the biggest problem facing you now? How does this relate to your symptoms, your past experiences, your hopes for yourself?

- What are some of the ways you have mastered important problems in the past?

- Is there anyone who thinks you can get better, deal with your problems? How does he or she see the situation? 
- If you could deal with this problem more effectively, how might your life be different?

- Do think there is a reason that you have this problem?

The student writes up the patient's life story and reviews it with a supervising psychiatrist or resident. (The preparatory materials include a template for the write up and several samples). The next, and crucial step is for the student to go over the written account of the patient's story with the patient face to face. This step is both the most anxiety provoking for the student, and the one that drives home the importance of identifying and reinforcing sources of hope and empowerment in the complex and often tragic life stories that psychiatric inpatients have to tell.

\section{Evaluation of the therapeutic communication exercise}

David Garrison at the University of Rochester has published a qualitative analysis of the students' evaluation of the exercise as implemented in that setting ${ }^{6}$. The evaluation of the exercise in our setting is based solely on students' answers to a required form presented at the end of the rotation. This is not a well standardized instrument, and only impressionistic, qualitative results are available.

All students do the exercise, for which they are accountable on their logs of required experiences. Roughly two thirds of them report that doing the patient interview and providing feedback to the patients was worthwhile. In five years, less than a handful have reported that they thought it was harmful to a patient, or to their ability to relate to patients.

Representative negative comments: "My attempt at the therapeutic exercise was not very successful. Unfortunately, I picked a very difficult patient who was quite talkative and any attempts to refocus the conversation were futile".

"Patients I have encountered are very sensitive about their mental sickness. One schizophrenic patient became very upset when she read my description of her as having "disorganized thoughts".

Representative positive comments: "It was interesting to watch the patient respond to what I was saying. They really wanted answers to the reasons they were feeling certain ways and felt relieved when there was an explanation given".

"It was probably more useful to me than to the patient - to understand my ability to "hear" the patient as he or she wants to be heard and to respond accordingly".

"It was useful to me and my patient. It helped make a more therapeutic alliance".

\section{Discussion}

The post-Flexner consensus about what is important or essential in medicine is eroding in the face of rapid advances in knowledge and technology that blur the boundaries of what should be taught at what level. Medical education in the future must emphasize concepts rather than facts.

Recognizing demoralization and learning ways to improve patients' adaptation to their conditions and to the conditions of medical care should be one of the foundational concepts in medical education. As a dynamic dimension of illness that expresses itself in many ways, demoralization cannot simply be described in a lecture and later recognized and addressed when it appears. A case based 
approach that includes preparation, implementation and reflection is far better suited to the task. The exercise described above can be adapted to many settings and cultural contexts. It may help future providers of medical care to move from learning to treat diseases that accidently crop up in patients to the more elusive goal of becoming professionals able to help whole people who have fallen ill.
4. Frank JD, Frank JB. Persuasion and Healing: A Comparative Study of Psychotherapy, $3^{\text {rd }}$ edition. Baltimore: JHU Press; 1991.

5. Garrison D, Frank J. The Narrative Exercise: Introduction to Therapeutic Communication in the Psychiatry Clerkship; 2010. MedEdPORTAL. http://services.aamc.org/ 30/mededportal/servlet/s/segment/mededportal/?subid=7765 (retrieved Feb, 2011).

6. Garrison D, Lyness J, Frank J, Epstein RM. Qualitative analysis of medical student impressions of a narrative exercise in the third year psychiatry clerkship. Acad Med 2011; 86(1): 85-89.

\section{References}

1. American Psychiatric Association. Diagnostic and Statistical Manual IV-TR. Washington, DC: Am Psychiat Pub, Inc; 2000 .

2. UMDMJ Center for Teaching Excellence. Active Learning: Case Based Learning; 2006. http://cte.umdnj.edu/ active_learning/active_case.cfm retrieved Feb, 2011.

3. Barrows HS, Tamblyn R. Problem based learning: An approach to medical education. Springer Series on Medical Education, V 1. New York: Springer Pub Co; 2006.

\author{
Corresponding author: \\ Julia B. Frank, MD \\ Department of Psychiatry and Behavioral Sciences \\ George Washington University School of Medicine and \\ Health Sciences \\ 2120 L Street NW 600 \\ Washington, DC 20037 \\ Phone: 202-741-2865; 202-741-2891 (fax) \\ E-mail: jfrank@mfa.gwu.edu
}

